\title{
Modelling and Investigation of a Hybrid Thermal Energy Harvester
}

\author{
Todor Todorov ${ }^{1, *}$, Nikolay Nikolov ${ }^{1}$, Georgi Todorov ${ }^{2}$ Yanko Ralev ${ }^{1}$ \\ ${ }^{1}$ Technical University - Sofia, Faculty of Industrial Technology, Department of Theory of Mechanisms and Machines, 1797, \\ Sofia, Bulgaria \\ ${ }^{2}$ Technical University - Sofia, Faculty of Industrial Technology, Dept. of Technology of Machine Tools and Manufacturing, \\ 1797, Sofia, Bulgaria
}

\begin{abstract}
The presented paper deals with dynamical and experimental investigations of a hybrid energy harvester containing shape memory alloy (SMA) wire and elastic cantilever with piezoelectric layer. The SMA wire periodically changes its temperature under the influence of a heated plate that approaches and moves away from the SMA wire. The change of SMA wire length causes rotation of the hot plate. The plate is heated by a heater with constant temperature. The repeated SMA wire extensions and contractions bend the piezoelectric cantilever which generates electric charges. The shape memory effect is presented as a temperature approximation of the Young's modulus. A dynamical model of the energy harvester is created and some analytical investigations are presented. With the help of an experimental setup the acceleration, the force, the temperature, and the output voltage have been measured. The theoretical results are validated experimentally. Some conclusions are made about the best performance of the energy harvester.
\end{abstract}

\section{Introduction}

Energy harvesting devices convert otherwise unused ambient energy into small but useful levels of electrical energy. The most utilized energy sources are solar energy, mechanical vibration energy, and thermal energy. The utilization of waste heat is one of the most important fields of the energy harvesting. The currently mostly used methods for harvesting a mid to low grade thermal energy can be divided into thermal to direct electrical, and thermal-to-mechanical-to-electrical ones.

The thermal to electrical energy converters include thermionic, pyroelectric, and Seebeck generators, alkali metal thermal-to-electric converters, Johnson thermoelectrochemical converter, Johnson electrochemical heat pipe, and infrared photovoltaic harvesters [1-4]. Pyroelectric converters rely on the property of certain crystalline materials to generate an electrical potential when subjected to a temperature-time gradient $[5,6]$. Seebeck thermoelectric generators produce electricity by two different types of semiconductor materials when their junction is exposed to a temperature difference [7].

Thermomechanical heat engines are based on Stirling, Brayton, and Rankie thermodynamic cycles [812]. Thermoacoustic heat engines, thermomagnetic engines, hydride heat generators can be divided into two groups: traveling wave, and standing wave devices [1315]. Thermomagnetic engines rely on the change of magnetization of certain materials that occurs with change in temperature [16-18].
Shape memory alloy (SMA) are materials that allow relatively large strain about $8 \%$ when deformed and they completely recover their original shape when heated [19].

SMA engines transform thermal energy by SMAs using change of the temperature of water or air flow. Depending of type of the motion SMA engines can be divided into SMA engines with continuous and oscillating motion. SMA engines with continuous motion convert the reciprocating linear motion of an SMA actuator into continuous rotary motion [20-22]. Oscillating SMA engines consist of SMA elastic element (cantilever, bridge, wire) which moves between two end positions corresponding to heated and cooled state [23].

All above mentioned engines convert thermal-tomechanical energy and they could be the first part of thermal-to-mechanical-to-electrical converter. For the second part mechanical-to-electrical the well-known piezoelectric, magnetic, and electrostatic converters could be applied. Such energy harvesters with two different type converters are called hybrid or combined energy harvesters. Namli end Taya present Piezo-SMA composite for thermal energy harvesting under fluctuating temperature. Strain induced in the SMA material causes stress in the piezoelectric material, inducing electric charge by direct piezoelectric effect [24]. A remote light energy harvesting and actuation hybrid system, comprising SMA wire, attached to a piezoelectric bimorph cantilever, generates electric energy when wire is heated by a pulse laser [25]. A

Corresponding author: tst@.tu-sofia.bg 
thermal energy harvester with SMA/piezoelectric laminated composite consisting of $30 \mu \mathrm{m}$ thick $\mathrm{Ti}_{50} \mathrm{Ni}_{25} \mathrm{Cu}_{25}$ ribbons and piezoelectric layer of Micro Fiber Composite (MFC) induces electric charge under temperature fluctuations [26].

The aim of the present paper is to model and investigate a new SMA-piezoelectric energy harvester (SMA-PEH) consisting of SMA wire and piezoelectric cantilever. The energy source of the SMA-PEH is a heater with constant temperature. The constant temperature of the heater is delivered to the SMA wire as fluctuating temperature with the help of a simple vibrating mechanical system, driven by the SMA wire.

\section{Design concept of SMA-PEH}

In Figure 1(a) a schematic description of the proposed SMA-PEH in the beginning of SMA wire heating is presented. The SMA wire 1 is strained by cantilever 3 with piezoelectric bimorph 2. A brass plate 4, which is heated by heater 5 , touches the SMA wire 1 , which starts to heat as well. Figure 1 (b) illustrates the SMA-PEH with hot SMA wire. When the SMA wire gets hot, it changes its crystallographic structure from martensite to austenite, and it contracts. The free end of the cantilever moves. The deformation of the cantilever induces electric charge in the piezoelectric bimorph. At the same time by the limiters 6 the hot SMA wire rotates the brass plate 4 about the heat source 5 and heating of the SMA wire is off. Meanwhile, SMA wire cooling leads to austenite-to-martensite transformation, which decreases the SMA stiffness. The SMA wire strains again under the force action of the cantilever and the brass plate rotates counterclockwise to its position shown in Figure 1 (a). The motion of the free end of the cantilever deforms piezoelectric layer and a second electric charge is induced. Then all the processes described above are repeated.

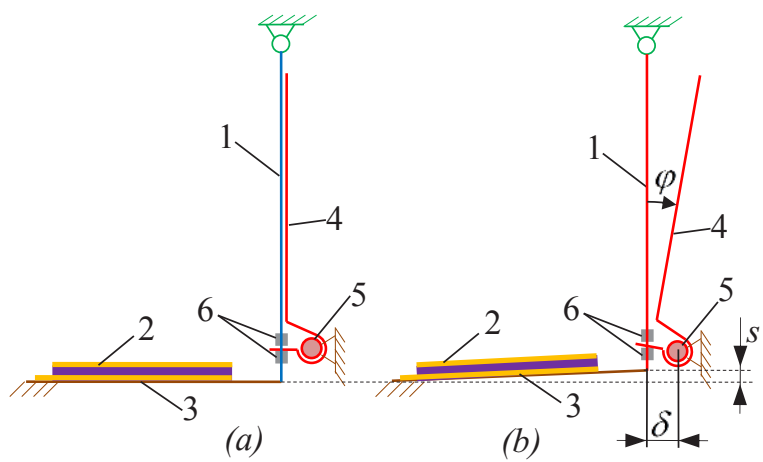

Fig. 1. Schematic representation of the thermal energy system: (a) SMA wire is cold (b) SMA wire is heated.

\section{Dynamical modelling of SMA-PEH}

Let consider the deformations of the piezoelectric cantilever and of the SMA wire shown in Figure 2.

The segments $\mathrm{CD}$ and $\mathrm{AE}$ are the initial undeformed states of the SPF wire and the cantilever when not connected (Figure 2a). At the beginning the cantilever and the SMA wire have a common point $\mathrm{A}$. In this

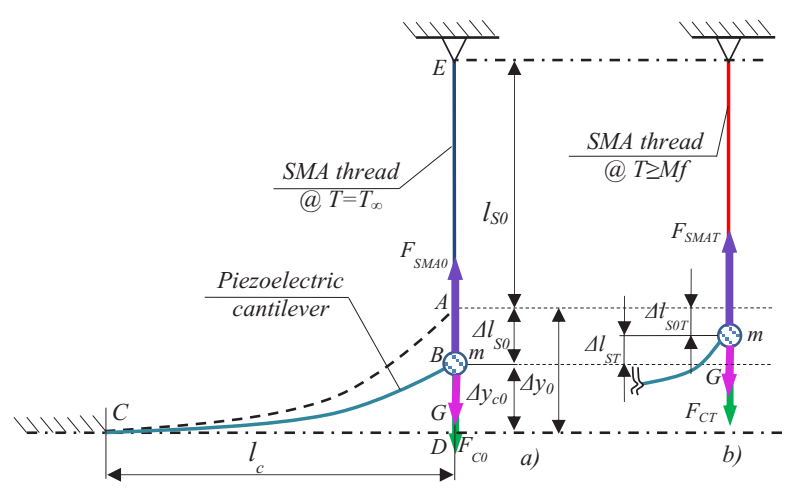

Fig. 2. Deformations of the SMA wire and of the piezoelectric cantilever.

state, the free cantilever end deformation is $\Delta y_{0}$, and the SMA wire length is $l_{S 0}$. At the equilibrium position, corresponding to point $\mathrm{B}$, the deformation of the free cantilever end decreases to $\Delta y_{c 0}$ and the SMA wire deformation is $\Delta l_{S 0}$. The force $F_{c 0}$ at the free cantilever end can be expressed as

$$
F_{c 0}=k_{c} \Delta y_{c 0},
$$

where

$$
k_{c}=\frac{3 E_{c} I}{l_{c}^{3}}
$$

is the stiffness of the cantilever, $E_{c}$ is the Young modulus of cantilever material, $l_{c}$ is the length of the cantilever. The cross-sectional moment of the cantilever is $I=\frac{w h^{3}}{12}$ , where $w$ is the width and $h$ is the thickness of the cantilever. The equilibrium force $F_{S O}$ in the SMA wire is presented in the form

$$
F_{S 0}=\frac{E_{M} A}{l_{S 0}} \Delta l_{S 0}
$$

where $E_{M}$ is the Young's modulus for starting martensite temperature of nitinol, and $A$ is the cross-section area of the SMA wire, $d$ is the wire diameter. We assume that at point B a generalized mass $m$ with weight $G$ also acts as

$$
G=m g
$$

From the equilibrium of the forces described by (1), (3), and (4) follows the equation

$$
k_{c} \Delta y_{e}+m g=\frac{E_{M} A}{l_{S 0}} \Delta l_{S 0} .
$$

Taking into account that the deformation $\Delta y_{0}$ is initially given according to Figure 2 (a) we can write

$$
\Delta y_{0}=\Delta l_{S 0}+\Delta y_{c 0} \text {. }
$$

Combining two above equations into a system, we can express

$$
\begin{aligned}
& \Delta y_{c 0}=\frac{\Delta y_{0} E_{M} A-l_{S 0} m g}{E_{M} A+k_{c} l_{S 0}}, \\
& \Delta l_{S 0}=\frac{\left(\Delta y_{0} k_{c}+m g\right) l_{S 0}}{E_{M} A+k_{c} l_{S 0}} .
\end{aligned}
$$

In Figure 2 b) it is described the static load of the considered mechanical system, when the wire is heated to an arbitrary temperature over the final martesite 
temperature $M f$ and less than the final austenite temperature $A f$. The wire heating leads to structure changes that increase the Young's modulus. The wire hardens, and it decreases it's elastic deformation. The distance $\Delta l_{S}$, that is the reduction of the elastic deformation due to the heating, we call temperature deformation. Expressing the equilibrium of the vertical forces in this case, we can write

$$
F_{C T}+G=F_{S M A T}
$$

where the force of the cantilever at temperature deformation is

$$
F_{C T}=k_{c}\left(\Delta y_{C}+\Delta l_{S}\right)
$$

and the force in the SMA wire is

$$
F_{S M A T}=\frac{E(T) A}{l_{S 0}} \Delta l_{S 0 T}
$$

Taking in account that

$$
\Delta y_{0}=\Delta l_{S 0 T}+\Delta y_{c 0}+\Delta l s
$$

substituting (4), (10), and (11) into (9) and combining with (12) we can express

$$
\begin{gathered}
\Delta l_{S}=\frac{A l_{S 0}\left(k_{c} \Delta y_{0}+g m\right)\left[E(T)-E_{M}\right]}{\left(A E_{M}+k_{c} l_{S 0}\right)\left[A E(T)+k_{c} l_{S 0}\right]}, \\
\Delta l_{S 0 T}=\frac{\left(k_{c} \Delta y_{0}+m g\right) l_{S 0}}{E(T) A+k_{c} l_{S 0}} .
\end{gathered}
$$

We assume that the Young's modulus $E(T)$ is a temperature function presented in the form

$$
E=2 E_{M}+\frac{E_{A}-E_{M}}{2} \tanh \frac{T-A s}{k_{E}}
$$

where

$$
k_{E}=\frac{E_{A}-E_{M}}{A f-M f}
$$

$E_{A}$ is SMA Young's module for austenite structure, $A f$ is the final austenite temperature, $M f$ is the final martensite temperature, and $A s$ is the start austenite temperature. We neglected hysteresis because the strain of the SMA wire is smaller than $0,3 \%$.

The graph of the approximated Young's modulus is shown in Figure 3.

With the help of the Young's modulus approximation (15) we can express the straintemperature graph shown in Figure 4.

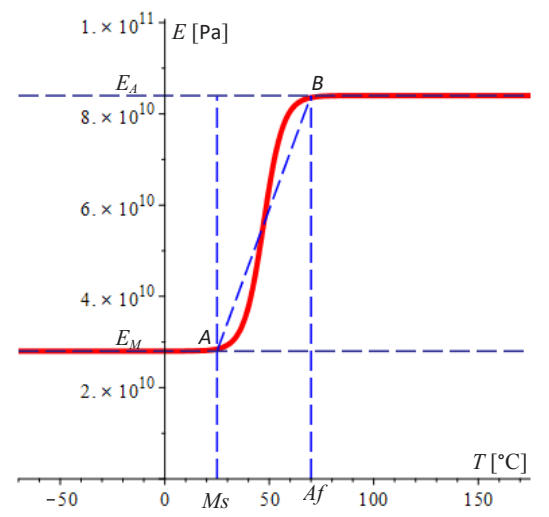

Fig. 3. Young's modulus approximation.
The analysis shows that the maximum temperature deformation does not recover the total elastic strain of the SMA wire. This is so because at the austenite phase there is an elastic strain, which can vanish only when there is no stress in the SMA wire.

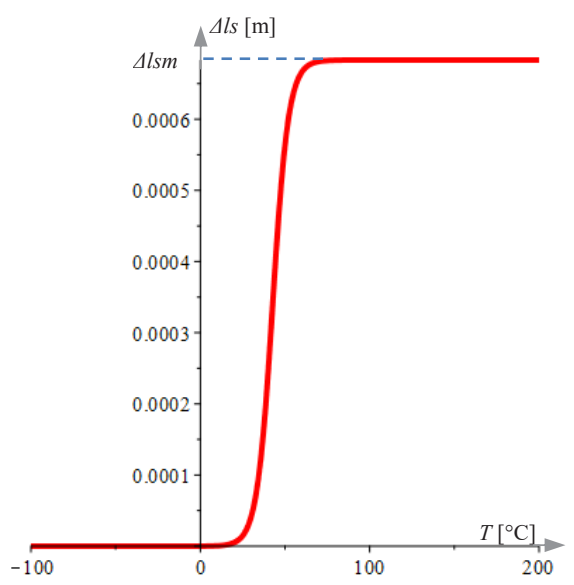

Fig. 4. Temperature deformation vs. time.

For creating a dynamical model we assume that there is a lumped mass at the free end of the cantilever. We denote the position of this mass as $y$. The force $F_{S M A}$ of the SMA wire that exerts on the mass is

$$
F_{S M A}=\left\{\begin{array}{cc}
\frac{E(T) A\left(\Delta l_{S 0}-y+\Delta y_{c 0}\right)}{l_{S 0}} & y<\Delta y_{c 0}+\Delta l_{S 0} \\
0 & y \geq \Delta y_{c 0}+\Delta l_{S 0}
\end{array}\right.
$$

In the above expression we take into account that the force $F_{S M A}$ is unidirectional and it can only strain the SMA wire.

From Figure 1 we see that the angle $\varphi$ is constrained to be positive only, because the negative value is possible if the plate penetrates into the SMA wire. This consideration leads to the formula

$$
\varphi=\left\{\begin{array}{cc}
\arctan \frac{y-\Delta y_{e}}{b} & y-\Delta y_{e}>0 \\
0 & y-\Delta y_{e} \leq 0
\end{array}\right.
$$

For the heat transfer between hot plate and SMA wire the following relationship is assumed:

$$
T_{h p}=T_{r}+\frac{T_{h}}{2}\left(1+\cos \frac{\pi \varphi}{\varphi_{m}}\right),
$$

where $T_{h}$ is the temperature of the hot plate, $T_{h p}$ is the temperature which is transferred to the SMA wire, $\varphi_{m}$ is the maximum angle of the hot plate, and $T_{r}$ is an experimentally determined temperature.

The unidirectional action of the SMA wire can change the damping coefficient of the system in the form

$$
\beta=\left\{\begin{array}{cc}
\beta_{S M A}+\beta_{c} & y<\Delta y_{c 0}+\Delta l_{S 0} \\
\beta_{c} & y \geq \Delta y_{c 0}+\Delta l_{S 0}
\end{array}\right.
$$

where $\beta_{S M A}$ is the damping coefficient of the SMA wire and $\beta_{c}$ is the damping coefficient of the cantilever.

On the basis of the above assumptions a dynamical model was created, presented in the view 


$$
\mid \begin{gathered}
m \frac{d^{2} y}{d t^{2}}+\beta \frac{d y}{d t}+k_{c} y=F_{S M A} \\
\rho A c_{p} \frac{d T}{d t}-h_{c} A_{c}\left\{T_{\infty}-T+T_{h p}[\varphi(y)]\right\}=0
\end{gathered}
$$

where $m$ is the lumped mass, $\rho$ is the density of the SMA wire material, $c_{p}$ is the specific heat, $h_{c}$ is the heat convective transfer coefficient, $A_{c}$ is the circumferential area per unit length of the wire, and $T_{\infty}$ is the room temperature.

In the above system the first equation describes the force balance, and the second equation describes the temperature processes of the energy harvester [28, 29].

The system (21) is solved numerically by Euler's implicit method of $5^{\text {th }}$ order.

The solution of the system (16) shows that the amplitude of the displacement is modulated and it increases in the transition period. The same is the behavior of the acceleration (Figure 5).

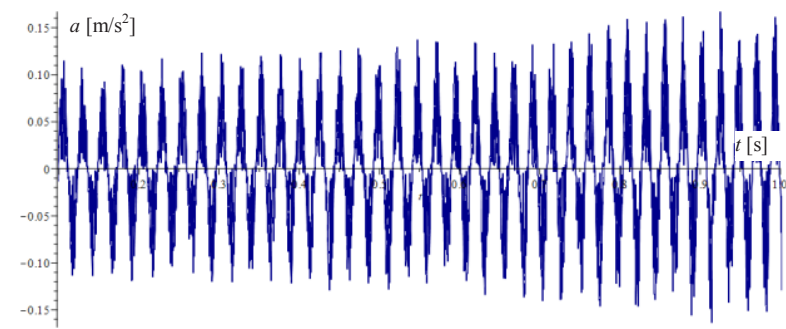

Fig. 5. Acceleration $a$ of the free cantilever end vs. time $t$.

The temperature of the SMA increases modulated as it is presented in Figure 6. There is an equilibrium temperature at $62^{\circ} \mathrm{C}$ corresponding to the temperature of the heater in a middle position.

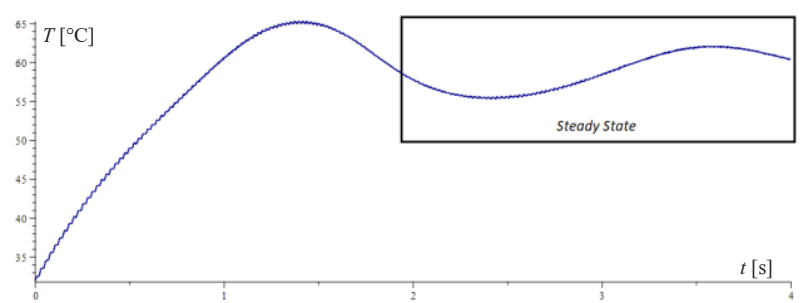

Fig. 6. Temperature of the SMA wire vs. time.

Form the obtained above solution we can conclude that in the behavior of the mechanical and of the thermal parameters two types of vibrations are observed: The first one - proportional to the temperature time constant, and the second one - equal to the natural frequency of the mechanical part. According to Donald J. L. [29] the temperature time constant $\tau$ is determined by the relation

$$
\tau=\frac{\rho A c_{p}}{h_{c} A_{c}} .
$$

Neglecting the damping, the mechanical natural frequency can be calculated by the formula

$$
\Omega_{\Sigma}=\sqrt{\frac{k_{c}+k_{s m a}}{m}},
$$

where

$$
k_{\text {sma }}=\frac{E(T) A}{l_{S 0}}
$$

is the stiffness of the SMA wire.

Formula (23) shows that besides the amplitude modulation there is an additional frequency modulation. The amplitude modulation is due to the thermal shortening of the SMA wire. The frequency modulation is due to the stiffness change of the wire when heated. Both modulations are due to the thermal change but they reflect two different properties of the SMA material: shortening and stiffening.

\section{Design concept of the experimental setup}

The evaluation setup for investigation of SMA-PEH is shown in Figure 7. The sensor signals of the SMA-PEH 1 are processed by a Data Acquisition System (DAQ) 2. The sensors and heater are powered up by two rectifiers 3,4 and a thermal regulator 5. The DAQ output enters into a laptop 6, where the experiment is controlled by a LabVIEW program.

In Figure 8 the SMA-PEH with the sensors is presented. A piezorestive MEMS force sensor 1 measures the longitudinal force in SMA wire. The hot plate 2 rotates about the fixed axis of a heater 3 , which is a part of soldering iron. The acceleration of the cantilever beam 4 along vertical axis is measured by a three-axis MEMS accelerometer 5.

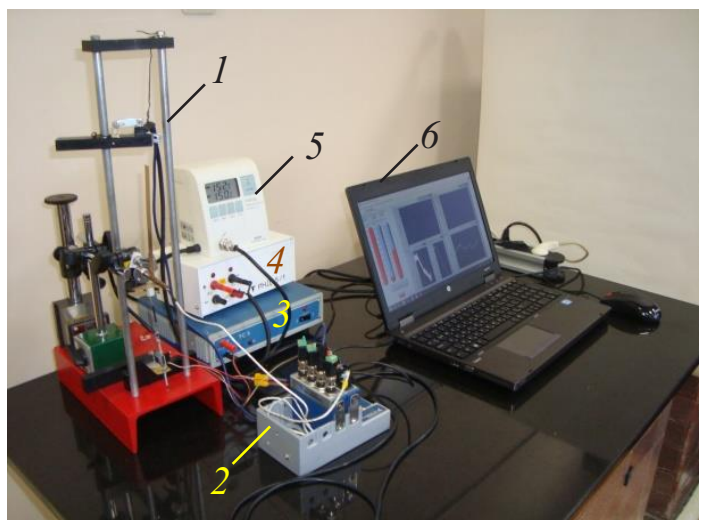

Fig. 7. Evaluation setup of the hybrid SMA-PEH: 1. SMAPEH; 2. DAQ; 3.4. Rectifiers; 5. Thermal regulator. 6. Laptop.

For obtaining the necessary data of the temperature three thermocouples are used. The thermocouple 6 measures the heater temperature. Thermocouple 7 is used to measure the room temperature. In order to increase the frequency response of the thermocouple for the SMA wire, a thermocouple type-T with a small diameter of $100 \mu \mathrm{m}$ is used, as shown in Figure 9. 


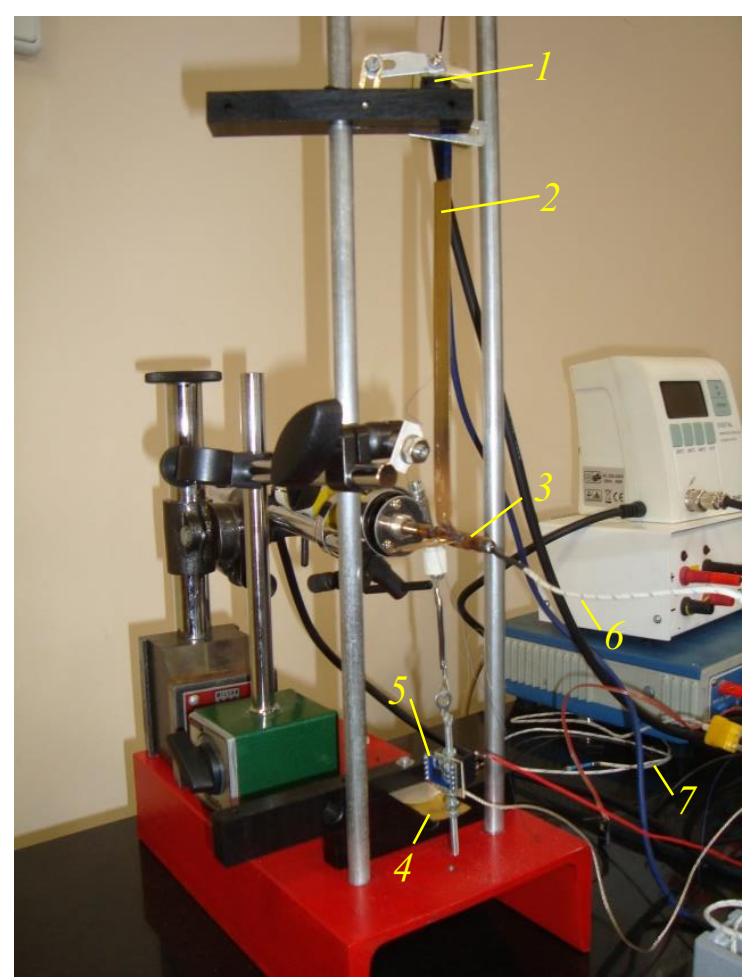

Fig. 8. Closeup of SMA-PEH: 1. Force sensor; 2. Hot plate; 3. Heater; 4. Piezoelectric cantilever; 5. Accelerometer. 6. Thermocouple for the heater, 7 Thermocouple for the room temperature.

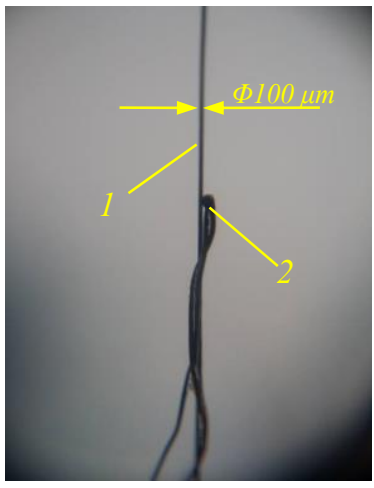

Fig. 9. The SMA wire and its thermocouple: 1.SMA wire, 2. Thermocouple.

\section{An experimental validation interpretation of the model}

By the evaluation setup, described above, consequently series of experiments were performed. Results of one such an experiment are shown in Figures 13-16.

In Figure 10 the experimental results for the acceleration of the free end of the cantilever are presented. We see that the output of the accelerometer is a periodic oscillation with stable behavior. Compared to the model acceleration (Figure 5), we can register a relative good fit with respect to the amplitude, shape and frequency.

The experimentally obtained temperature change of the SMA wire is shown in Figure 10. Compared to the theoretical result from Figure 6, for the steady state period a good fit is demonstrated.

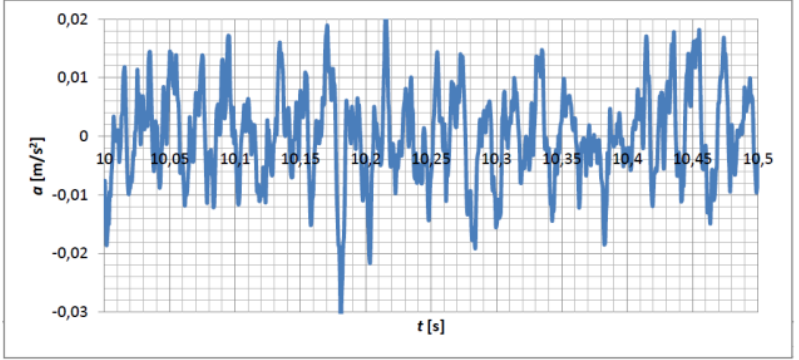

Fig. 10. Experimental acceleration $a$ of the free cantilever end vs. time $t$.

The measured output voltage is presented in Figure 12. Maximum amplitude of about $50 \mathrm{mV}$ is registered at load resistance of $10 \mathrm{k} \Omega$ which corresponds to $0,5 \mu \mathrm{W}$ peak-to-peak power.

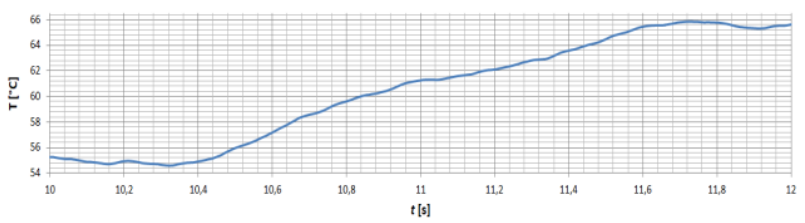

Fig. 11. Experimentally determined temperature $T$ of the SMA wire for the steady state of 2 seconds.

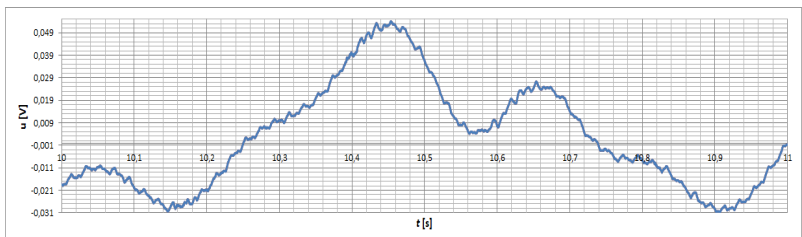

Fig. 12. Measured output $u$ voltage of the energy harvester

The best efficiency of the energy harvester we expect to have when the frequency of the mechanical and of the thermal oscillations coincide. As it was already clarified, the energy harvester has a variable frequency. Therefore, the condition for frequency coincidence is possible to be satisfied only for one value of the temperature of the SMA wire. The frequency equalizing in the considered device requires a wire with a very small diameter and the smallest possible lumped mass.

\section{Conclusions}

A new design of a combined thermoelectric energy harvester, containing SMA wire and piezoelectric flexible member for generation of electric energy from constant heat source is presented.

A dynamical model of the energy harvester is built and investigated.

With the help of an experimental setup model, an experimental validation is performed.

The comparison between model results and experiments indicates a good validation of temperature during the steady state period, and in terms of acceleration satisfactory coincidences of amplitudes and vibration periods are obtained.

The condition for the best performance of the energy harvester according to parametric resonant systems is the coincidence of twice frequency and the system's natural 
mechanical frequency. Because the two frequencies vary with temperature change, this condition can only be achieved for a certain temperature value of the SMA wire.

The presented work is carried out and funded under contract DUNK-01/3 between National Science Fund, MES of $\mathrm{RB}$ and R\&D Sector of TU-Sofia.

\section{References}

1. Percy S. Knight C. McGarry S. Post A. Moore T. Cavanagh K., Thermal Energy Harvesting for Application at MEMS Scale, Springer, (2014)

2. Hernqvist, K.G. Kanefsky M. Norman F.H., Thermionic Energy Converter. RCA Rev. 19, 2 (1958)

3. Sievers R.K. Ivanenok J.F. Hunt T.K. Alkali metal thermal to electric conversion, Mechanical Engineering; 117, 10 (1995)

4. Johnson L.G. Muller J.R. Johnson reversible engine, US Patent, US7160639 B2, (2007)

5. Hossain A. Rashid M.H. Pyroelectric detectors and their applications. IEEE Trans Ind Appl. 27 (1991)

6. Whatmore R.W. Pyroelectric devices and materials. Rep Prog Phys. 49 (1986)

7. Liang G. Zhou J. Huang X. Analytical model of parallel thermoelectric generator, Applied Energy 88, 12 (2011)

8. Patterson D.Ee Jamison K,D, Durrett M. CVD diamond based miniature stirling cooler International cryocooler conference (2007)

9. Nakajima N. Ogawa K. Fujimasa I. Study on micro engines-miniaturizing Stirlingengines for actuators and heatpumps. Sens Actuators 20, 75-82, (1989)

10. Rosa R.J. Characteristics of a closed Brayton cycle piston engine, IEEE Western Canada conference on computer, power and communications systems in a rural environment, pp 153-159, (1991)

11. Johnston J.R. Evaluation of expanders for use in a solar-powered Rankine Cycle Heat Engine. Doctoral dissertation, Ohio State University, pp 1-138, (2001)

12. Cho J. Lin C. Richards C Demonstration of an external combustion micro-heat engine. Proc Combust Inst 32, 3099-3105 (2009)

13. Pierens M. Thermeau J.P. Le Pollès T. Duthil P. Experimental characterization of a thermoacoustic travelling-wave refrigerator. International conference on fluid mechanics, heat transfer and thermodynamics (2011)

14. Ceperley P.H. A Pistonless Stirling Engine-the Traveling Wave Heat Engine.J Acoust Soc Am 66, 1508-1513, (1979)
15. Swift G.W. Thermoacoustic Engines, J Acoust Soc Am 841145 (1988)

16. Solomon D (1991) Design of a thermomagnetic generator. Energy Convers Manage 31:157-173.

17. Hsu C-J, Sandoval SM, Wetzlar KP, Carman GP (2011) Thermomagnetic conversion efficiencies for ferromagnetic materials. J Appl Phys 110:123923123927.

18. Bulgrin KE, Ju YS, Carman GP, Lavine AS (2009) A coupled thermal and mechanical model of a thermal energy harvesting device. ASME 2009 International Mechanical Engineering Congress \& Exposition. Lake Buena Vista, Fl, pp 327-335

19. Otsuka K. Wayman C.M. Shape memory materials, $1^{\text {st }}$ edn. Cambridge University Press, Cambridge (1998)

20. Schiller E.H. Heat Engine Driven by Shape Memory Alloys:Prototyping and Design, Master thesis, Virginia Polytechnic Institute (2002)

21. Hochstein P.A. Some Engineering Parameters for a Nitinol Engine Design, Proc. of the Nitinol Heat Engine Conf., Silver Spring, Maryland, (1978)

22. Sandoval D.J. Nitinol Belt Engine, Proc. of the Nitinol Heat Engine Conf., Silver Spring, Maryland, (1978)

23. Kahn H, Huff MA, Heuer AH The TiNi shapememory alloy and its applications for MEMS. J Micromech Microeng 8 213-221 (1998)

24. Namli O.C. Taya M. Design of piezo-SMA composite for thermal energy harvester under fluctuating temperature, J. Appl. Mech., 78, 8 (2011)

25. Avirovik D. Kumar A. Bodnar R.J. Priya S. Remote light energy harvesting and actuation using shape memory alloy-piezoelectric hybrid transducer, Smart Mater. Struct., 22, 6 (2013)

26. Lebedev G.A. Gusarov B.V. Viala B. Delamare J. Cugat O. Lafont T. Zakharov D.I. Thermal energy harvesting using shape memory alloy piezoelectric composite, in Proc. 16th Int. Solid-State Sens. Actuators Microsyst. Conf. (Transducers'11), Beijing, China, pp. 669-670 (2011)

27. Ralev Y. Todorov T. Modelling of Shape Memory alloys and experimental setup for their investigation, CAx Technologies, 4, (2016) 46-51,

28. Bhargaw H. N. Ahmed M., Sinha P. Thermoelectric behaviour of NiTi shape memory alloy, Trans. Nonferrous Met. Soc. China, 23, (2013) 2329-2335

29. Donald J. L. Engineering analysis of smart material systems, Hoboken, New Jersay: John Wiley \& Sons, Inc, (2007) 333-350 